\title{
Proposal for a Simple Polarization Converter based on Integrated Optical Ion Exchanged Waveguide
}

\author{
Pengfei Wang ${ }^{1,2}$, Gilberto Brambilla ${ }^{1}$, Yuliya Semenova ${ }^{2}$, Qiang Wu ${ }^{2}$, Jie Zheng $^{3}$, Gerald Farrell ${ }^{2}$ \\ ${ }^{1}$ Optoelectronics Research Centre, University of Southampton, Southampton, SO17 1BJ, UK \\ ${ }^{2}$ Photonics Research Center, School of Electronic and Communications Engineering, Dublin Institute of Technology, Kevin \\ Street, Dublin 8, Ireland \\ ${ }^{3}$ State Key Laboratory of Integrated Optoelectronics, College of Electronic Science and \\ Engineering, Jilin University, Changchun 130012, P. R. China \\ *E-mail: pw3y09@orc.soton.ac.uk
}

Keywords: Optical polarization converter, glass waveguide, ion exchange, liquid crystal.

\begin{abstract}
A simple, compact, low-cost electro-optic polarization converter based on a nematic liquid crystal (LC) sandwiched between two ion-exchanged glass channel waveguides is proposed. A three-dimensional (3D) fully-vectorial (FV) finite difference beam propagation method (FDBPM) is used to simulate this optical device. The performance of the proposed polarization converter is analyzed numerically at zero and $5 \mathrm{~V}$ applied voltage for the case of strong anchoring of LC molecules to the surface of the waveguide. The polarization conversion efficiency is $\sim 65 \%$ at a wavelength of $1550 \mathrm{~nm}$. The total length of the directional coupler structure based converter is only $724 \mu \mathrm{m}$.
\end{abstract}

\section{Introduction}

Numerous photonic integrated circuits (PICs), such as planar lightwave circuits and photonic crystal circuits, have been recently investigated, mainly driven by the development of optical communications and optical sensing. They have excellent characteristics, such as low fabrication cost, compact size and high scalability by comparison to bulk optical devices.

Liquid crystal (LC) materials have also been widely used in photonics due to their high birefringence, superior electrooptical properties and a relatively fast response time in the order of milliseconds. Recently several authors have reported the use of liquid crystals combined with PICs to fabricate various photonic devices, e.g., integrated optical switches, polarization splitters, modulators etc [1-4].

For bulk optical devices based on liquid crystals, a range of accurate models for the simulation of light behaviour has been developed in recent years. For example, matrix methods, such as the Jones or the Berremann matrix methods are popularly employed for theoretical prediction. When the device size is comparable to the operating wavelength, other numerical methods, such as finite-difference time domain method (FDTD) or beam propagation method (BPM) are essential to simulate the light behaviour within a PIC device that combines a LC material with an isotropic dielectric structure. In this paper, theoretical modelling and numerical simulations of an integrated liquid crystal photonic device based on ion-exchanged waveguides are carried out using a three-dimensional fully-vectorial finite-difference beam propagation method (FDBPM). Based on this an integrated compact electro-optic polarization converter is presented.

Ion-exchanged glass waveguide based PICs have been extensively investigated due to the numerous advantages they offer, such as very low propagation loss, low birefringence, low coupling loss with singlemode optical fibres and a relatively simple fabrication technique. A number of configurations for integrated ion exchanged glass waveguide based PICs have been proposed [5, 6]. However, to the best of our knowledge, no investigation on an ion exchange waveguide combined with LC has been reported. In this paper an integrated LC-ion exchanged polarization converter is proposed, designed and simulated. The proposed optical polarization converter consists of a LC layer sandwiched between ion exchanged channel waveguide substrates. The application of an electric field above the threshold value in the direction perpendicular to the LC layer with a positive dielectric anisotropy causes changes in the LC molecular orientation from a planar alignment at the surface of the waveguide to a homeotropic alignment (perpendicular to the surfaces of the ion-exchanged waveguides). This results in polarization-dependent changes of the light propagating along the ion-exchanged channel waveguides. In our simulations strong anchoring of the LC molecules to the surface of the ion-exchanged waveguide is assumed. The light propagation in the integrated device is simulated and characterized in the absence of an electric field and under the influence of an external applied voltage. Results indicate that surface anchoring conditions for the liquid crystal at the surface of the ion-exchanged waveguide are the key issue in realizing the expected polarization conversion efficiency; the interaction coupling length also indicates a strong mode confinement induced by the LC layer in the waveguides gap. The proposed integrated device benefits from the simple and compact structure and from its ease of fabrication. 


\section{Proposed configuration of the device and theoretical modelling tools}

\subsection{Proposed device configuration}

The schematic structure of the proposed device is shown in Fig. 1. A liquid crystal layer is sandwiched between the surfaces of ion-exchanged glass waveguides with deposited ITO electrodes. The whole device can be regarded as a directional coupler containing a liquid crystal cell, with the planar lightwave circuit acting as a substrate. In simulations two possible liquid crystal molecular orientations are considered: planar alignment along the $x$-axis and a homeotropic alignment along the $y$-axis as a result of the electric field applied to the LC layer.

The separation between the two waveguides, i.e. the thickness of the LC layer, and the length of the directional coupler are assumed to be $10 \mu \mathrm{m}$ and $1000 \mu \mathrm{m}$, respectively. Here the nematic LC E7 is chosen with the ordinary and extraordinary refractive indices of $n_{o}=1.5$ and $n_{e}=1.7$ at the wavelength of $\square=1550 \mathrm{~nm}$. The value of the refractive index for the ion exchanged waveguide $n_{c o}$ is chosen between $n_{o}$ and $n_{e}$. The refractive index of the Schott SF11 glass substrate is assumed to be 1.7434@1550 nm and the maximum difference between the waveguide and the substrate refractive indices is chosen as 0.049 . The width of the photomask for the ion exchange is $3 \mu \mathrm{m}$.

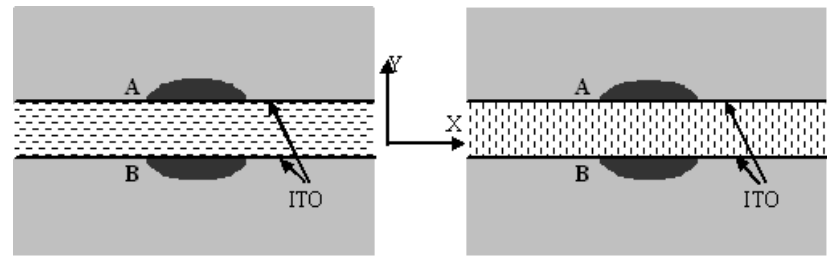

(a)

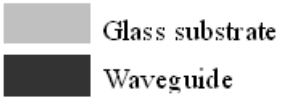

(b)

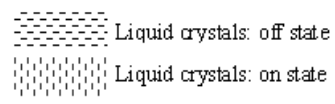

Fig. 1. Schematic configuration of an integrated polarization converter.

The operating principle of this polarization converter is based on the fact that due to the birefringence of the liquid crystal, the TE and TM modes of the waveguide have different index profiles in the liquid crystal area adjacent to the waveguide and consequently have different propagation characteristics. In configuration (a) in Fig. 1, the TE mode (the polarization direction is defined in the $\mathrm{x}-\mathrm{Z}$ plane) is confined within the LC layer, while the TM mode (the polarization direction is in the $\mathrm{y}-\mathrm{z}$ direction) propagates in both the waveguide and LC areas due to the presence of strong guiding. In configuration (b), which can be achieved by applying a threshold electric field to the LC layer, the TM mode is confined within the LC layer and the TE mode propagates in both the waveguide and the LC layer near the surface of the waveguide.

To carry out the theoretical design and numerical analysis, a supermode solution for an ion-exchanged waveguide combined with a birefringent material and corresponding fully-vectorial beam propagation method are briefly described below.

\subsection{Theoretical modelling tools}

The wave equation in a birefringent dielectric is

$$
\nabla \times \nabla \times \vec{E}-k^{2} \hat{\varepsilon}(x, y, z) \vec{E}=0
$$

where $k=2 \pi / \lambda_{0}$ and $\lambda_{0}$ is the wavelength in free-space. In a birefringent medium such as the layer of liquid crystal, $\hat{\varepsilon}$ is an optical tensor. For configuration (a): $\varepsilon_{x x}=n_{e}^{2}$, $\varepsilon_{y y}=\varepsilon_{z z}=n_{o}^{2}, \varepsilon_{m n}=0$ (where $m, n=x, y, z$, and $m \neq n$ ), while for configuration (b): $\varepsilon_{y y}=n_{e}^{2}, \varepsilon_{x x}=\varepsilon_{z z}=n_{o}^{2}$, $\mathcal{E}_{m n}=0 \quad$ (where $m, n=x, y, z$, and $m \neq n$ ). For both configurations, in the waveguide core area the refractive index profile of the ion-exchanged waveguide is adequately described by $\varepsilon_{x x}=\varepsilon_{y y}=\varepsilon_{z z}$ and $\varepsilon_{m n}=0$, while in the surrounding substrate region, $\varepsilon_{x x}=\varepsilon_{y y}=\varepsilon_{z z}=n_{s u b}^{2}$ and $\varepsilon_{m n}=0$. The $\mathrm{x}$ - and $\mathrm{y}$-components of the electric field satisfy

$$
\begin{gathered}
-\frac{\partial^{2}}{\partial z^{2}}\left[\begin{array}{l}
E_{x} \\
E_{y}
\end{array}\right]=\left[\begin{array}{ll}
P_{x x} & P_{x y} \\
P_{y x} & P_{y y}
\end{array}\right]\left[\begin{array}{c}
E_{x} \\
E_{y}
\end{array}\right] \\
\text { where } P_{x x} E_{x}=\frac{\partial}{\partial x}\left[\frac{1}{\varepsilon_{z z}} \frac{\partial}{\partial x}\left(\varepsilon_{x x} E_{x}\right)\right]+\frac{\partial^{2} E_{x}}{\partial y^{2}}+k^{2}\left[\left(\varepsilon_{x x}-n_{0}^{2}\right) E_{x}\right] \\
P_{x y} E_{y}=\frac{\partial}{\partial x}\left[-\frac{\partial E_{y}}{\partial y}+\frac{1}{\varepsilon_{z z}} \frac{\partial}{\partial y}\left(\varepsilon_{y y} E_{y}\right)\right] \\
P_{y x} E_{x}=\frac{\partial}{\partial y}\left[-\frac{\partial E_{x}}{\partial x}+\frac{1}{\varepsilon_{z z}} \frac{\partial}{\partial x}\left(\varepsilon_{x x} E_{x}\right)\right] \\
P_{y y} E_{y}=\frac{\partial^{2} E_{y}}{\partial x^{2}}+\frac{\partial}{\partial y}\left[\frac{1}{\varepsilon_{z z}} \frac{\partial}{\partial y}\left(\varepsilon_{y y} E_{y}\right)\right]+k^{2}\left[\left(\varepsilon_{y y}-n_{0}^{2}\right) E_{y}\right]
\end{gathered}
$$

Based on these BPM equations, modelling of the beam behaviour for two polarization states in the ion exchanged waveguide covered by a liquid is presented in the following section.

Modelling of the ion exchange distribution is also needed. The nonlinear diffusion equation for ion-exchange is [6-10]

$$
\frac{\partial}{\partial t} C=\frac{D_{A}}{1-\alpha C}\left[\nabla^{2} C+\frac{\alpha}{1-\alpha C}(\nabla C)^{2}\right]
$$

where $C$ is the normalized ion concentration of $\mathrm{A}$ ions. $\alpha=1-D_{A} / D_{B}, D_{A}$ and $D_{B}$ are the self-diffusion coefficients for $\mathrm{A}$ ions and $\mathrm{B}$ ions respectively. The $\mathrm{A}$ and $\mathrm{B}$ ions are assumed as $\mathrm{Ag}$ and $\mathrm{Na}$ ions, respectively. In this model, 
$D_{A}=5.1 \times 10^{-6}$ and $\alpha=0.87$ are chosen [7]. Ion-exchange and annealing times are assumed to be 20 and 35 minutes, respectively. The length of the waveguide is $1000 \mu \mathrm{m}$. The width of the waveguide on the photomask is $3 \mu \mathrm{m}$. The simulated contour plots of full-vectorial eigenmodes of the ion-exchanged glass waveguide are presented in Fig.2 (a) and Fig.2 (b).

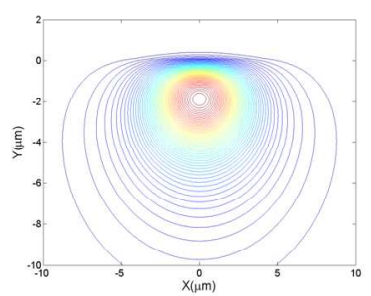

(a)

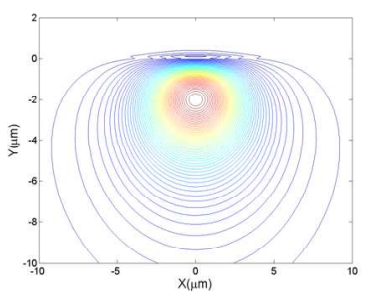

(b)
Fig. 2. Full-vectorial eigenmodes of an ion-exchanged glass waveguide: (a) $E_{x}$ of the quasi-TE mode; (b) $E_{y}$ of the quasi-TM mode.

The eigenmode solution of a 3D waveguide with a LC covering layer is essential element of the modelling of the proposed integrated LC devices. In this numerical example, the channel ion-exchanged glass waveguide presented above is chosen. The LC used in the above example is chosen as the covering layer with planar alignment (OFF state - no external electric field applied). With the 3D FV FDBPM developed above, the $\mathrm{E}_{\mathrm{x}}$ and $\mathrm{E}_{\mathrm{y}}$ components for the quasi-TE and quasiTM modes are presented in Fig. 3(a) and (b), respectively.

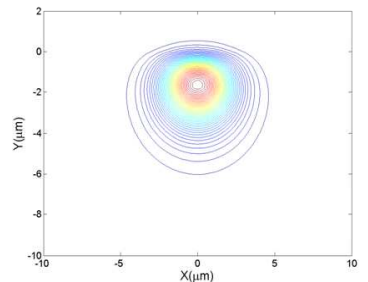

(a)

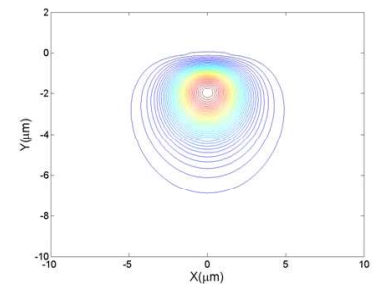

(b)
Fig. 3. Full-vectorial eigenmodes of an ion-exchanged glass waveguide covered by LC in the absence of electric field (OFFstate): (a) $\mathrm{E}_{\mathrm{x}}$ of the quasi-TE mode; (b) $\mathrm{E}_{\mathrm{y}}$ of the quasi-TM mode.

\section{Performance of integrated polarization converter}

In the numerical example, the thickness of the LC layer is assumed to be $10 \mu \mathrm{m}$. It is known that in practice, while the LC molecules in the middle of the LC layer re-orient along the field [11], application of the electric field does not change the alignment of the LC molecules near the surface of the electrodes. In this paper strong anchoring alignment is chosen for the theoretical analysis. For the chosen nematic LC (E7 from Merck), the corresponding parameters are $k_{11}=11.7 \times 10^{-12} \mathrm{~N}, k_{22}=9.0 \times 10^{-12} \mathrm{~N}, k_{33}=19.5 \times 10^{-12} \mathrm{~N}$, $\varepsilon_{/ /}=19.2, \varepsilon_{\perp}=5.3$ ).

Light propagation in the device shown in Fig. 1 is investigated with a 3D model for two cases: (1) off state (no applied voltage); (2) the applied voltage is $5 \mathrm{~V}$ with a strong surface anchoring.

For both cases, the eigenmodes of waveguide B calculated by the above example (as shown in Fig. 2(a) and 2(b)) are chosen to be the input fields. With this 3D FV FDBPM, the propagation of the input fields along the device is calculated and the simulation results are shown in Fig. 4(a) and 4(b) for both cases. Transmission losses in waveguide B for both TE and TM modes along the propagation distance are presented in Fig. 5. From Figs. 4 (a) and 4(b), it is possible to see that both TE and TM modes are basically confined in waveguide B and the TM mode is coupled to the TE mode while propagating along the device.

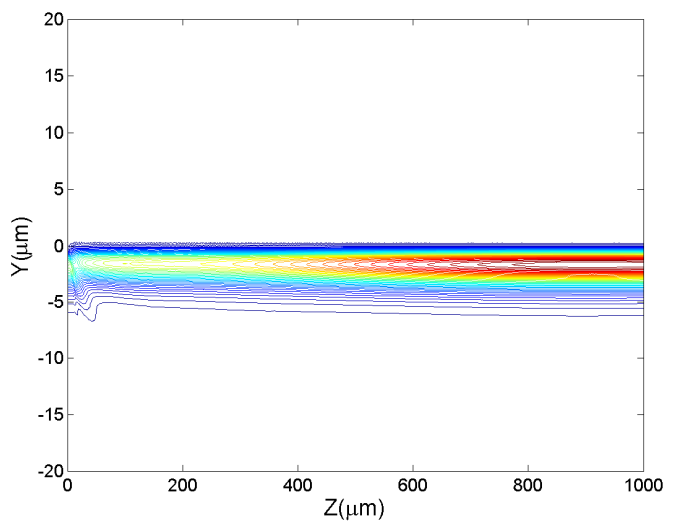

(a)

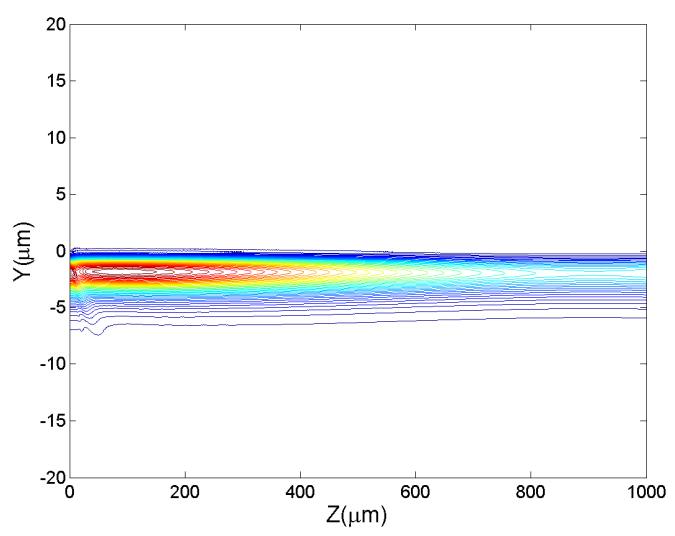

(b)

Fig. 4. Contour plots of (a) TE mode; (b) TM mode along the propagation distance within the device.

To better characterize the transmission performance of the proposed LC device, the transmission losses of both TE and $\mathrm{TM}$ modes at $5 \mathrm{~V}$ in the case of strong anchoring are simulated with the model based on a 3D FV FDBPM for anisotropic materials. As shown in Fig. 5, for the case of OFF state, both TE and TM losses remain almost constant as the propagation distance increases; for the case of strong anchoring with applied voltages of $5 \mathrm{~V}$, the TE loss decreases and the TM loss increases as the propagation distance increases; the absolute change of TM loss is higher than that of TE. The polarization conversion efficiency is circa $65 \%$ at the operating wavelength of $1550 \mathrm{~nm}$. The results in Fig. 5 
also suggest that for this device to work as an optical polarization converter, the coupling length should be at the propagation distance of $724 \mu \mathrm{m}$. The increase in attenuation for TM loss is due to the strong interaction between the substrate surface and the liquid crystal molecules near the surface of ion-exchanged waveguide. This value of TE to TM attenuation ratio (the extinction coefficient) can be valuable in the practical design of a more efficient polarization converter.

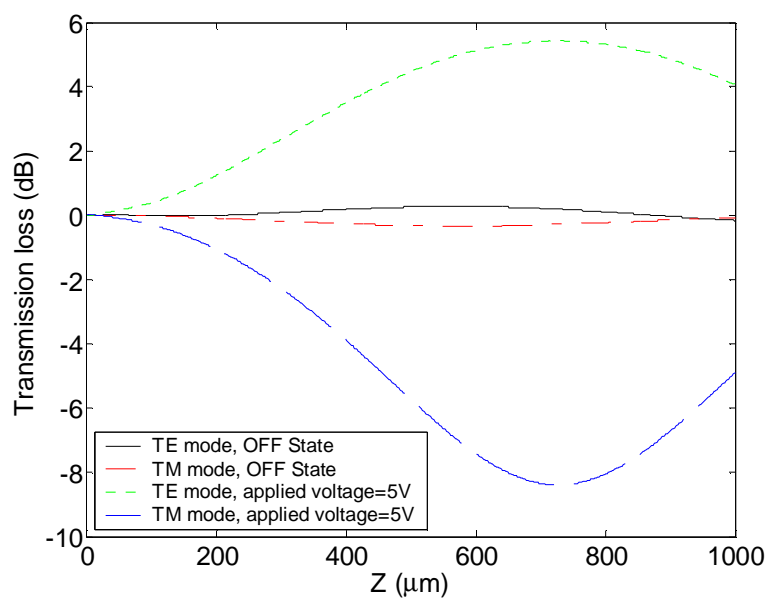

Fig. 5. Transmission losses in glass waveguide B versus propagation distance for the proposed polarization converter.

\section{Conclusion}

In this paper, light propagation within an LC sandwiched by two ion-exchanged glass waveguides based optical polarization converter has been investigated. The three-dimensional full-vector finite-difference beampropagation-method for an anisotropic medium has been presented, allowing beam propagation within the integrated optical polarization converter to be modelled. The effect of strong surface anchoring on the device performance has been demonstrated numerically. The polarization conversion efficiency is $\sim 65 \%$ at a wavelength of $1550 \mathrm{~nm}$. The total length of the directional coupler structure based converter is $724 \mu \mathrm{m}$. Further work is required to improve the efficiency of the device by changing the intensity of the external electrodes in the design. This would also significantly reduce the length of directional coupler and consequently minimize the size of the LC device.

\section{Acknowledgements}

Pengfei Wang is funded by the Irish Research Council for Science, Engineering and Technology, co-funded by the Marie-Curie Actions under FP7. Gilberto Brambilla gratefully acknowledges the Royal Society (London, UK) for his University Research Fellowship.

The research was partially supported by the National Natural Science Foundation of China (No. 60777038), the China-Ireland Science and Technology Collaboration Research Fund and the International cooperation project
(No.20070708-3) of Jilin Provincial Science \& Technology Department of China.

\section{References}

[1]. N. A. Riza and S. Yuan, "Reconfigurable wavelength add-drop filtering based on a Banyan network topology and ferroelectric liquid crystal fiber-optic switches", J. Lightwave Technol.17, 1575-1584 (1999).

[2]. K. Wu, J. Liu and Y. Chen, "Optical attenuator using polarization modulation and a feedback controller", US patent 5963291, 1999.

[3]. K. Hirabayashi and C. Amano, "Liquid-crystal polarization controller arrays on planar waveguide circuits", IEEE Photonics Technol. Lett. 14, 504-506 (2002).

[4]. Y. Semenova, Y. Panarin, G. Farrell and S. Dovgalets, "Liquid crystal based optical switches", Mol. Cryst. Liq. Cryst. 413, 2521-2534 (2004).

[5]. R. A. Betts, F. Lui and S. Dagias, "Wavelength and polarization insensitive optical splitters fabricated in $\mathrm{K}^{+} / \mathrm{Na}^{+}$Ion-exchanged glass", IEEE Photon. Technol. Lett., Vol. 2, No. 7, pp.481-483, (1990).

[6]. B. Buchold, C. Glingener, D. Culemann and E. Voges, "Polarization insensitive ion-exchanged array-waveguide grating multiplexers in glass", Fiber and Integrated Optics, Vol. 17, pp. 279-298, (1998).

[7]. B. R. West, P. Madasamy, N. Peyghambarian and S. Honkanen, "Modeling of ion-exchanged glass waveguide structures", J. Non-Crystalline Solids, Vol. 347, pp. 1826, (2004).

[8]. J. T. A. Carriere, J. A. Frantz, B. R. West, S. Honkanen and R. K. Kostuk, "Bend loss effects in diffused, buried waveguides”, App. Opt., Vol. 44, No. 9, pp. 1698-1703, (2005).

[9]. J. M. Castro, D. F. Geraghty, B. R. West and S. Honkanen, "Fabrication and comprehensive modelling of ion-exchanged Bragg optical add-drop multiplexers", App. Opt. Vol. 43, No. 33, pp. 6166-6173, (2004).

[10]. S. Yliniemi, B. R. West and S. Honkanen, "Ionexchanged glass waveguides with low birefringence for a broad range of waveguide widths", App. Opt., Vol. 44, No. 16, pp. 3358-3363, (2005).

[11]. M. P. Allen, "Molecular simulation and theory of liquid crystal surface anchoring", Molecular Physics, Vol. 96, No. 9, pp. 1391 - 1397, (1999). 